\title{
Derived neutrophil/lymphocyte ratio predicts gemcitabine therapy outcome in unresectable pancreatic cancer
}

\author{
REI SUZUKI ${ }^{1}$, TADAYUKI TAKAGI ${ }^{1}$, TAKUTO HIKICHI $^{2}$, NAOKI KONNO $^{1}$, \\ MITSURU SUGIMOTO ${ }^{1}$, KO WATANABE ${ }^{2}$, JUN NAKAMURA ${ }^{1}$, YUICHI WARAGAI ${ }^{1}$, \\ HITOMI KIKUCHI $^{1}$, MIKA TAKASUMI ${ }^{1}$, HIROSHI WATANABE ${ }^{1}$ and HIROMASA OHIRA ${ }^{1}$ \\ Departments of ${ }^{1}$ Gastroenterology and Rheumatology and ${ }^{2}$ Endoscopy, \\ School of Medicine, Fukushima Medical University, Fukushima 960-1295, Japan \\ Received February 16, 2015; Accepted March 15, 2016
}

DOI: $10.3892 / \mathrm{ol} .2016 .4381$

\begin{abstract}
As gemcitabine is a key anti-tumor agent for unresectable pancreatic ductal adenocarcinoma (PDAC), it is important to predict the outcomes of gemcitabine chemotherapy. The present study aimed to confirm whether the derived neutrophil-to-lymphocyte ratio (dNLR) is able to predict chemotherapy outcomes. To elucidate the role of dNLR in patients that underwent chemotherapy, the current study evaluated clinicopathological variables in 31 patients with unresectable PDAC treated with gemcitabine. The correlation between clinicopathological variables, and progression-free survival (PFS) and overall survival (OS) time were investigated. Univariate analysis revealed that there were no significant differences in PFS and OS as a function of age ( $<65$ vs. $\geq 65$ years), gender, tumor location (pancreas head vs. body/tail), tumor diameter ( $<23 \mathrm{vs} . \geq 23 \mathrm{~mm}$ ) or serum carbohydrate antigen $19-9$ concentration level $(<3,800$ vs. $\geq 3,800 \mathrm{U} / \mathrm{ml}$ ). However, disease stage (locally advanced vs. metastatic) and the dNLR ( $<2.5$ vs. $\geq 2.5)$ significantly affected PFS and OS. Multivariate analysis subsequently revealed that a $d N L R$ of $\geq 2.5$ was an independent prognostic factor for poor PFS $(\mathrm{P}=0.003)$ and $\mathrm{OS}(\mathrm{P}=0.026)$. In conclusion, data from the present study suggests that the pre-treatment dNLR is an independent prognostic factor to predict PFS and OS in patients with unresectable PDAC treated with gemcitabine. This indicates that dNLR has a potential role in stratifying patients that may benefit from gemcitabine therapy.
\end{abstract}

Correspondence to: Dr Rei Suzuki, Department of Gastroenterology and Rheumatology, School of Medicine, Fukushima Medical University, 1 Hikarigaoka, Fukushima 960-1295, Japan

E-mail: subaru@fmu.ac.jp

Key words: pancreatic carcinoma, chemotherapy, marker, neutrophil, lymphocyte

\section{Introduction}

Pancreatic ductal adenocarcinoma (PDAC) remains a worldwide healthcare dilemma (1). Due to the improved quality of imaging modalities and other diagnostic procedures, the annual incidence of PDAC has increased in various countries, being the fourth leading cause of cancer-associated mortality in the USA, with an overall 5-year survival rate of $\sim 10 \%$ (2). One of the standard treatments for PDAC is gemcitabine chemotherapy, as $\sim 80 \%$ of patients present with unresectable or metastatic disease (3). Clinical trials have demonstrated that several new chemotherapy regimens have superior anti-tumor efficacy compared with gemcitabine $(4,5)$. However, gemcitabine remains a key chemotherapeutic agent for palliative chemotherapy in patients with PDAC due to its moderate anti-tumor effect and limited adverse events. Numerous studies aiming to stratify patients using molecular markers, including human equilibrative nucleoside transporter 1 and ribonucleotide reductase regulatory subunit M1, have been conducted to determine candidates for whom gemcitabine treatment would be optimal (6-8). These molecular markers are reliable predictive markers for gemcitabine response in patients with PDAC, but typically require specialized equipment that is difficult to utilize in routine clinical practice.

Previous studies have revealed that tumor inflammation is important in carcinogenesis, cancer progression and chemotherapy resistance (9-11). Among various immunological processes, neutrophils and lymphocytes are reported to have a vital role in tumor inflammation and immunology. It has been demonstrated that the peripheral blood neutrophil-to-lymphocyte ratio (NLR), or more recently the derived NLR (dNLR), may be a reliable prognostic marker in various types of cancer, including PDAC (12-18), However, the use of the dNLR in predicting susceptibility to chemotherapy has not been evaluated. Therefore, the present study aimed to evaluate whether the dNLR is a reliable predictive marker for gemcitabine response in patients with unresectable PDAC. In addition, the efficacy of the dNLR was compared with other clinicopathological markers, including serum carbohydrate antigen (CA) 19-9 level. We consider it clinically important to identify patients that will not have a robust response to gemcitabine and to identify those that should instead be treated with highly 
intensive chemotherapy regimens, in order to maximize the therapeutic window.

\section{Patients and methods}

The present retrospective cohort study reviewed data from patients diagnosed with unresectable PDAC, including locally advanced and metastatic disease, at Fukushima Medical University (Fukushima, Japan) between September 2006 and January 2014. Patients with histopathologically confirmed PDAC were included, whereas those who were assumed to have PDAC based on imaging findings or serum tumor marker levels were excluded from the study. Additionally, patients with rare primary pancreatic neoplasms, including acinar cell carcinoma or neuroendocrine carcinoma, were excluded. All patients were chemotherapy-naïve prior to undergoing $>2$ cycles of gemcitabine treatment at $1,000 \mathrm{mg} / \mathrm{m}^{2}$ on days 1,8 and 15 of a 28-day cycle. Adverse events were graded according to the National Cancer Institute Common Toxicity Criteria version 4.0, within the Cancer Therapy Evaluation Program (http://dctd.cancer .gov/ProgramPages/CTEP/, accessed on May 6, 2013). If severe hematological toxicity (>grade 3) was observed, the dose was reduced to $800 \mathrm{mg} / \mathrm{m}^{2}$; if patients were unable to tolerate this reduced dose, the dose was administered biweekly. Patients were treated with gemcitabine alone until disease progression or unacceptable toxicity (grade $>3$ adverse events in biweekly treatment protocol) was observed. When gemcitabine treatment failed, additional treatment was administered based on the physicians' decision. All clinicopathological data utilized in the present study were measured immediately prior to the initial chemotherapy session. The dNLR was calculated using the following formula: Neutrophil count / (white blood cell count - neutrophil count) (19). The present study was approved by the institutional review board (IRB) of Fukushima Medical University (Fukushima, Japan) as a retrospective cohort study (protocol number, 2286). The IRB of Fukushima Medical University waived the need for written informed patient consent due to the retrospective non-interventional nature of the study.

Statistical analysis. The progression-free survival (PFS) and overall survival (OS) time were calculated from the date of histological diagnosis to the date of disease progression and mortality, respectively, since no complete response was observed in any of the patients of the present cohort. Receiver operating curve analysis was conducted to determine ideal cut-off values of poor prognosis for the following continuous variables: Tumor diameter, serum CA 19-9 level and the dNLR. The association of each clinicopathological parameter [age, gender, tumor location, maximum tumor diameter, disease stage (locally advanced unresectable PDAC or PDAC with metastatic lesions), serum CA 19-9 level and dNLR] with PFS and OS were investigated. Survival analysis was performed using the Kaplan-Meier method with log-rank test in univariate analysis. Multivariate Cox regression analysis using forward stepwise selection was performed to determine the effect of clinicopathological variables on survival time. Statistical analysis was performed using SPSS (version 21; IBM SPSS, Chicago, IL, USA). $\mathrm{P}<0.05$ was considered to indicate a statistically significant difference.
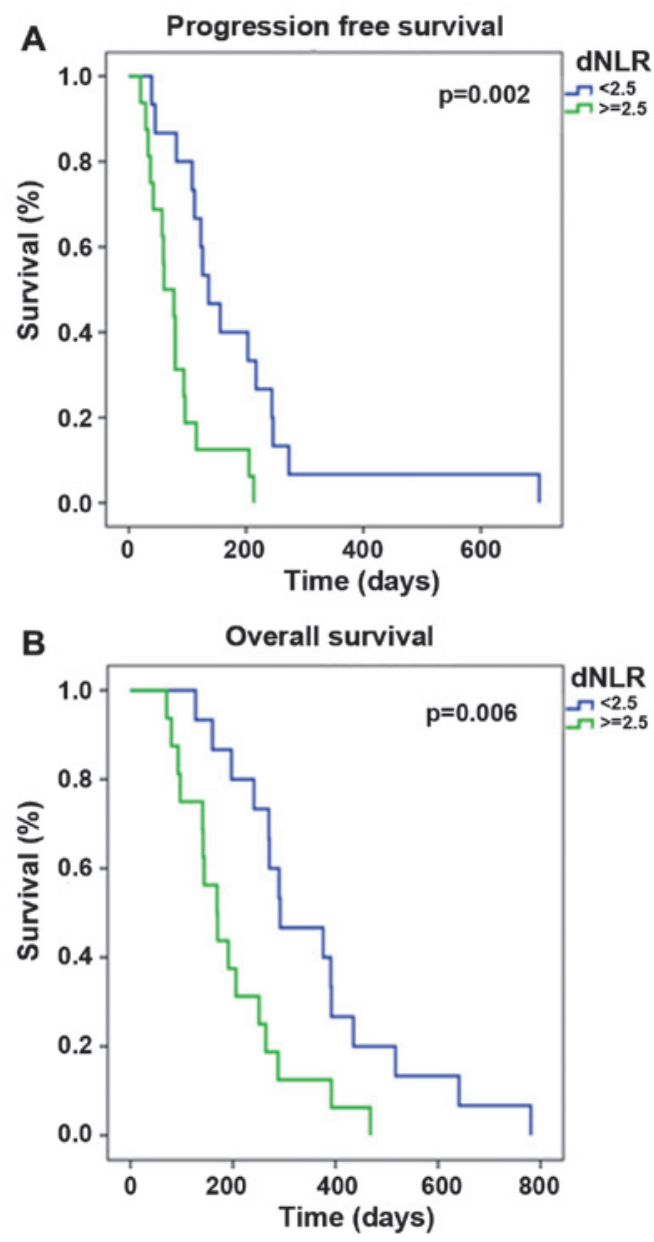

Figure 1. Kaplan-Meier analysis of (A) progression-free and (B) overall survival according to a high $(\geq 2.5)$ vs. low $(<2.5)$ dNLR, indicating significant associations. dNLR, derived neutrophil-to-lymphocyte ratio.

\section{Results}

Between September 2006 and January 2014, 243 patients were diagnosed with PDAC. Among them, 31 patients who met the inclusion criteria were included in the present retrospective analysis. The majority of patients were excluded because of loss to follow-up. Clinical patient characteristics are indicated in Table I. Briefly, the cohort included 17 male and 14 female patients with a median age of 61 years (range, 49-75 years). The median PFS and OS times were 96 days (range, 20-700 days) and 251 days (range, 71-781 days), respectively.

The ideal cut-off values to predict the clinical outcome of continuous variables were tumor diameter of $23 \mathrm{~mm}$, serum CA 19-9 level of 3,800 U/ml and dNLR of 2.5.

Univariate analysis revealed that there were no significant differences in PFS and OS time as a function of age ( $<65$ vs. $\geq 65$ years), gender (male vs. female), tumor location (head of the pancreas vs. body and tail of the pancreas), tumor diameter ( $<23$ vs. $\geq 23 \mathrm{~mm})$ or serum CA 19-9 level $(<3,800$ vs. $\geq 3,800 \mathrm{U} / \mathrm{ml}$ ). By contrast, disease stage (locally advanced vs. metastatic) and the dNLR ( $<2.5$ vs. $\geq 2.5)$ significantly affected the PFS $(\mathrm{P}=0.02$ and $\mathrm{P}=0.002$, respectively) and $\mathrm{OS}$ $(\mathrm{P}=0.006$ and $\mathrm{P}=0.006$, respectively; Table $\mathrm{I})$. The dNLR was an independent prognostic factor for PFS and OS time according to multivariate analysis $(\mathrm{P}=0.003$ and $\mathrm{P}=0.026$, 
Table I. Univariate analysis of clinicopathological variables according to PFS and OS time.

\begin{tabular}{|c|c|c|c|c|c|}
\hline \multirow[b]{2}{*}{ Variable } & \multirow[b]{2}{*}{ Total patients, n (\%) } & \multicolumn{2}{|l|}{ PFS time } & \multicolumn{2}{|l|}{ OS time } \\
\hline & & Median survival, days & P-value & Median survival, days & P-value \\
\hline Age, years & & & 0.874 & & 0.458 \\
\hline$<65$ & $18(58.1)$ & 145.1 & & 291.0 & \\
\hline$\geq 65$ & $13(41.9)$ & 114.5 & & 254.6 & \\
\hline Gender & & & 0.338 & & 0.320 \\
\hline Male & $17(54.8)$ & 108.3 & & 253.5 & \\
\hline Female & $14(45.2)$ & 161.6 & & 302.7 & \\
\hline Location & & & 0.356 & & 0.140 \\
\hline Head & $14(45.1)$ & 104.1 & & 223.7 & \\
\hline Body/tail & $17(54.9)$ & 155.7 & & 318.5 & \\
\hline Diameter, mm & & & 0.053 & & 0.210 \\
\hline$<23$ & $4(12.9)$ & 214.7 & & 403.5 & \\
\hline$\geq 23$ & $27(87.1)$ & 120.2 & & 256.8 & \\
\hline Stage & & & 0.021 & & 0.006 \\
\hline Locally advanced & $8(25.8)$ & 239.5 & & 422.3 & \\
\hline Metastatic & $23(71.2)$ & 95.1 & & 224.7 & \\
\hline CA 19-9, U/ml & & & 0.121 & & 0.134 \\
\hline$<3,800$ & $21(67.7)$ & 150.4 & & 298.1 & \\
\hline$\geq 3,800$ & $10(32.3)$ & 94.5 & & 228.8 & \\
\hline dNLR & & & 0.002 & & 0.006 \\
\hline$<2.5$ & $15(48.4)$ & 187.3 & & 358.7 & \\
\hline$\geq 2.5$ & $16(51.6)$ & 80.9 & & 197.9 & \\
\hline
\end{tabular}

PFS, progression-free survival; OS, overall survival; CA 19-9, carbohydrate antigen 19-9; dNLR, derived neutrophil-to-lymphocyte ratio.

Table II. Multivariate analysis of clinicopathological variables according to PFS and OS time.

\begin{tabular}{lcr}
\hline Variable & Hazard ratio $(95 \% \mathrm{CI})$ & P-value \\
\hline PFS & & \\
Location & & 0.026 \\
$\quad$ Head & $2.50(1.16-5.5)$ & \\
$\quad$ Body and tail & & 0.003 \\
dNLR & 1 (reference) & \\
$<2.5$ & $0.28(0.13-0.65)$ & \\
$\geq 2.5$ & & $<0.001$ \\
OS & & \\
Location & 1 (reference) & \\
Head & $3.68(1.49-9.05)$ & \\
Body and tail & & 0.022 \\
Stage & 1 (reference) & \\
Locally advanced & $0.27(0.09-8.31)$ & \\
Metastatic & & \\
dNLR & 1 (reference) & \\
$<2.5$ & $0.41(0.19-0.90)$ & \\
$\geq 2.5$ & & \\
\hline
\end{tabular}

PFS, progression-free survival; OS, overall survival; CI, confidential interval; dNLR, derived neutrophil-to-lymphocyte ratio. respectively; Table II) and Kaplan-Meier survival analysis $(\mathrm{P}=0.002$ and $\mathrm{P}=0.006$, respectively; Fig. 1$)$.

\section{Discussion}

To the best of our knowledge, there have been no studies investigating the association between the dNLR and tumor response to gemcitabine in patients with unresectable PDAC. The present study aimed to clarify the role of dNLR and other clinicopathological factors to predict PFS and OS time in patients with locally advanced unresectable or metastatic disease treated with gemcitabine.

With regard to gemcitabine, several molecular markers have been observed to predict response and prognosis. Human equilibrative nucleoside transporter 1, which regulates the intracellular uptake of gemcitabine into cancer cells, is a well-established molecular marker that is able to predict susceptibility to gemcitabine or prognosis in patients with pancreatic cancer following treatment $(6,20,21)$. Other molecular markers, including serum tumor markers carcinoembryonic antigen and CA 19-9, tumor tissue Notch 3 mRNA expression levels, and serum interleukin (IL)-6 and IL-1 $\beta$ levels, have been proposed as good markers to predict response to gemcitabinen in PDAC patients (22-24). However, various of the above markers of gemcitabine response have not been routinely utilized in clinical practice due to excessive costs and technically challenging factors. 
Proctor et al developed the dNLR by utilizing the readily accessible clinical variables of white blood cell and neutrophil counts (19). Although dNLR was revealed to be as useful as NLR in predicting the prognosis of patients with breast and colorectal cancer, the usefulness of dNLR as a marker for predicting the prognosis of patients with PDAC could not be verified in that study, due to the limited number of PDAC patients included in the heterogeneous population analyzed by the authors (19). Subsequently, Absenger et al performed an external validation study of the dNLR on a large cohort of patients with PDAC and confirmed that the pre-treatment dNLR was an independent prognostic factor for the clinical outcome of patients with PDAC (16). Based on the aforementioned results, the present study further focused on the role of the pre-treatment dNLR to predict response to gemcitabine in patients with unresectable PDAC. A dNLR of $>2.5$ was an independent predictive marker of poor PFS and OS time, whereas the traditional predictive marker CA 19-9 did not exhibit any significance in predicting response or survival.

Newer chemotherapy regimens, such as FOLFIRINOX or gemcitabine plus nab-paclitaxel, demonstrate superior anti-tumor efficacy compared with gemcitabine $(4,5)$, although not all patients can tolerate these regimens. In a previous study, the incidence of severe neutropenia and thrombocytopenia was significantly higher in patients treated with FOLFIRINOX compared with those administered gemcitabine alone (45.7 vs. $21.0 \%$ and 9.1 vs. $3.6 \%$, respectively) (4). Similarly, grade 3 or 4 neutropenia and febrile neutropenia were more frequent when a combination of gemcitabine plus nab-paclitaxel was administered compared with gemcitabine alone (38 vs. $27 \%$ and 3.0 vs. $1.0 \%$, respectively) (5). However, a post-hoc analysis of the metastatic adenocarcinoma of the pancreas study revealed that the combination of gemcitabine plus nab-paclitaxel may contribute to longer OS compared with gemcitabine monotherapy, even in patients with high inflammation marker levels (for example, high NLR) (25). Taken together, the findings of previous studies and the present study suggest that patients with a low dNLR can be treated with gemcitabine monotherapy, whereas those with a high dNLR may require highly intensive regimens for disease management.

A limitation of the current study was that it was a single-center study with a limited number of patients; therefore, the results should be validated in a larger population across multiple clinical sites.

In conclusion, the pre-treatment dNLR appears to be an independent prognostic factor for predicting the OS and PFS time of patients with unresectable PDAC. In addition, the results indicate the potential role of the dNLR to stratify patients who should be treated with highly intensive regimens rather than gemcitabine alone.

\section{Acknowledgements}

The present study thanks Elsevier B.V. (Philadelphia, PA, USA) for their writing assistance.

\section{References}

1. Siegel R, Ma J, Zou Z and Jemal A: Cancer statistics, 2014. CA Cancer J Clin 64: 9-29, 2014.
2. Surveillance, Epidemiology, and End Results (SEER): Program Research Data (1973-2007), National Cancer Institute, DCCPS, Surveillance Research Program, Surveillance Systems Branch, 2010.

3. Sener SF, Fremgen A, Menck HR and Winchester DP: Pancreatic cancer: A report of treatment and survival trends for 100,313 patients diagnosed from 1985-1995, using the national cancer database. J Am Coll Surg 189: 1-7, 1999.

4. Conroy T, Desseigne F, Ychou M, Bouché O, Guimbaud R, Bécouarn Y, Adenis A, Raoul JL, Gourgou-Bourgade S, de la Fouchardiére C, et al: FOLFIRINOX versus gemcitabine for metastatic pancreatic cancer. N Engl J Med 364: 1817-1825, 2011.

5. Von Hoff DD, Ervin T, Arena FP, Chiorean EG, Infante J, Moore M, Seay T, Tjulandin SA, Ma WW, Saleh MN, et al: Increased survival in pancreatic cancer with nab-paclitaxel plus gemcitabine. N Engl J Med 369: 1691-1703, 2013.

6. Greenhalf W, Ghaneh P, Neoptolemos JP, Palmer DH, Cox TF, Lamb RF, Garner E, Campbell F, Mackey JR, Costello E et al: Pancreatic cancer hENT1 expression and survival from gemcitabine in patients from the ESPAC-3 trial. J Natl Cancer Inst 106: djt347, 2014.

7. Nakagawa N, Murakami Y, Uemura K, Sudo T, Hashimoto Y, Kondo $\mathrm{N}$ and Sueda T: Combined analysis of intratumoral human equilibrative nucleoside transporter 1 (hENT1) and ribonucleotide reductase regulatory subunit M1 (RRM1) expression is a powerful predictor of survival in patients with pancreatic carcinoma treated with adjuvant gemcitabine-based chemotherapy after operative resection. Surgery 153: 565-575, 2013.

8. Ashida R, Nakata B, Shigekawa M, Mizuno N, Sawaki A, Hirakawa K, Arakawa T and Yamao K: Gemcitabine sensitivity-related mRNA expression in endoscopic ultrasound-guided fine-needle aspiration biopsy of unresectable pancreatic cancer. J Exp Clin Cancer Res 28: 83, 2009.

9. Farrow B, Sugiyama Y, Chen A, Uffort E, Nealon W and Mark Evers B: Inflammatory mechanisms contributing to pancreatic cancer development. Ann Surg 239:763-771, 2004.

10. Lesina M, Kurkowski MU, Ludes K, Rose-John S, Treiber M, Klöppel G, Yoshimura A, Reindl W, Sipos B, Akira S, et al: Stat $3 /$ Socs 3 activation by IL-6 trans-signaling promotes progression of pancreatic intraepithelial neoplasia and development of pancreatic cancer. Cancer Cell 19:456-469, 2011.

11. Logsdon CD, Simeone DM, Binkley C, Arumugam T, Greenson JK, Giordano TJ, Misek DE, Kuick R and Hanash S: Molecular profiling of pancreatic adenocarcinoma and chronic pancreatitis identifies multiple genes differentially regulated in pancreatic cancer. Cancer Res 63:2649-2657, 2003.

12. Yamanaka T, Matsumoto S, Teramukai S, Ishiwata R, Nagai Y and Fukushima M: The baseline ratio of neutrophils to lymphocytes is associated with patient prognosis in advanced gastric cancer. Oncology 73: 215-220, 2007.

13. Chua W, Charles KA, Baracos VE and Clarke SJ: Neutrophil/lymphocyte ratio predicts chemotherapy outcomes in patients with advanced colorectal cancer. Br J Cancer 104: 1288-1295, 2011.

14. Lee S, Oh SY, Kim SH, Lee JH, Kim MC, Kim KH and Kim HJ: Prognostic significance of neutrophil lymphocyte ratio and platelet lymphocyte ratio in advanced gastric cancer patients treated with FOLFOX chemotherapy. BMC Cancer 13: 350, 2013.

15. Absenger G, Szkandera J, Pichler M, Stotz M, Arminger F, Weissmueller M, Schaberl-Moser R, Samonigg H, Stojakovic T and Gerger A: A derived neutrophil to lymphocyte ratio predicts clinical outcome in stage II and III colon cancer patients. Br J Cancer 109: 395-400, 2013.

16. Absenger G, Szkandera J, Stotz M, Postlmayr U, Pichler M, Ress AL, Schaberl-Moser R, Loibner H, Samonigg H and Gerger A: Preoperative neutrophil-to-lymphocyte ratio predicts clinical outcome in patients with stage II and III colon cancer. Anticancer Res 33: 4591-4594, 2013.

17. Martin HL, Ohara K, Kiberu A, Van Hagen T, Davidson A and Khattak MA: Prognostic value of systemic inflammation-based markers in advanced pancreatic cancer. Intern Med J 44: 676-682, 2014.

18. Szkandera J, Stotz M, Eisner F, Absenger G, Stojakovic T, Samonigg H, Kornprat P, Schaberl-Moser R, Alzoughbi W, Ress AL, et al: External validation of the derived neutrophil to lymphocyte ratio as a prognostic marker on a large cohort of pancreatic cancer patients. PLoS One 8: e78225, 2013.

19. Proctor MJ, McMillan DC, Morrison DS, Fletcher CD, Horgan PG and Clarke SJ: A derived neutrophil to lymphocyte ratio predicts survival in patients with cancer. Br J Cancer 107: 695-699, 2012. 
20. Nakano Y, Tanno S, Koizumi K, Nishikawa T, Nakamura K, Minoguchi M, Izawa T, Mizukami Y, Okumura T and Kohgo Y: Gemcitabine chemoresistance and molecular markers associated with gemcitabine transport and metabolism in human pancreatic cancer cells. Br J Cancer 96: 457-463, 2007.

21. Farrell JJ, Elsaleh H, Garcia M, Lai R, Ammar A, Regine WF, Abrams R, Benson AB, Macdonald J, Cass CE, et al: Human equilibrative nucleoside transporter 1 levels predict response to gemcitabine in patients with pancreatic cancer. Gastroenterology 136: 187-195, 2009.

22. Haas M, Heinemann V, Kullmann F, Laubender RP, Klose C, Bruns CJ, Holdenrieder S, Modest DP, Schulz C and Boeck S: Prognostic value of CA 19-9, CEA, CRP, LDH, and bilirubin levels in locally advanced and metastatic pancreatic cancer: Results from a multicenter, pooled analysis of patients receiving palliative chemotherapy. J Cancer Res Clin Oncol 139: 681-689, 2013.
23. Mitsunaga S, Ikeda M, Shimizu S, Ohno I, Furuse J, Inagaki M, Higashi S, Kato H, Terao K and Ochiai A: Serum levels of IL-6 and IL-1 $\beta$ can predict the efficacy of gemcitabine in patients with advanced pancreatic cancer. Br J Cancer 108: 2063-2069, 2013.

24. Eto K, Kawakami H, Kuwatani M, Kudo T, Abe Y, Kawahata S, Takasawa A, Fukuoka M, Matsuno Y, Asaka M and Sakamoto N: Human equilibrative nucleoside transporter 1 and Notch3 can predict gemcitabine effects in patients with unresectable pancreatic cancer. Br J Cancer 108: 1488-1494, 2013.

25. Goldstein D, El-Maraghi RH, Hammel P, Heinemann V, Kunzmann V, Sastre J, Scheithauer W, Siena S, Tabernero J, Teixeira L, et al: Nab-paclitaxel plus gemcitabine for metastatic pancreatic cancer: Long-term survival from a phase III trial. J Natl Cancer Inst 107: doi: 10.1093/jnci/dju413, 2015. 